\title{
MS42-P14 | Diffusion Mechanisms of Gas Adsorption by Porous Frameworks FROM SUB-SECOND SYNCHROTRON POWDER X-RAY DifFRACTION
}

Dovgaliuk, lurii (Swiss-Norwegian Beamlines at ESRF , Grenoble, FRA); Li, Xiao (Université Catholique de Louvain, Louvain-laNeuve, BEL); Dyadkin, Vadim (Swiss-Norwegian Beamlines at ESRF, Grenoble, FRA); Filinchuk, Yaroslav (Université Catholique de Louvain, Louvain-la-Neuve, BEL); Chernyshov, Dmitry (SNBL at the ESRF, Grenoble, FRA)

Nanoporous frameworks attract much attention as the potential materials for technological applications in diffusion-controlled processes such as selective guest adsorption and separation. Particular interest is payed for selective capture and separation of chemically inert $\mathrm{Kr}$ and Xe generated by nuclear fission [1]. Using advanced sub-second in situ powder X-ray diffraction (PXRD), three diffusion scenario have been evaluated from the isothermal kinetics adsorption by nanoporous $\gamma-\mathrm{Mg}\left(\mathrm{BH}_{4}\right)_{2}$ for a series of gases: $\mathrm{Ar}, \mathrm{Kr}$ and $\mathrm{Xe}$ [2]. The microscopic diffusion pathways and the activation barriers were rationalized from the macroscopic kinetic models and the crystal structure analysis. The lowest activation barrier has been estimated for the Ar atoms, which are smallest in the series. Consequently, these atoms equally easy diffuse through the intra- and interchannel apertures of $\gamma$ $\mathrm{Mg}\left(\mathrm{BH}_{4}\right)_{2}$ crystal structure. The diffusion of larger $\mathrm{Kr}$ atoms involves two activation energies: both intra- and interchannel barriers, which are higher than ones for Ar. Finally, the largest from the series Xe atoms diffuse only along 1-D channels with the highest activation barrier. The obtained kinetic characteristics are essential for the estimation of gas selectivity.

Therefore, sub-second PXRD offers unique information on the energetics and microscopic mechanisms of diffusion not accessible in macroscopic gravimetric and volumetric methods. This information relates solely to the crystalline fraction of material and can be compared with the atomistic models of diffusion.

[1] L. Chen et al. Nat. Mater. 2014, 13, 954-960;

[2] Y. Filinchuk et al. Angew. Chemie - Int. Ed. 2011, 50, 11162-11166. 